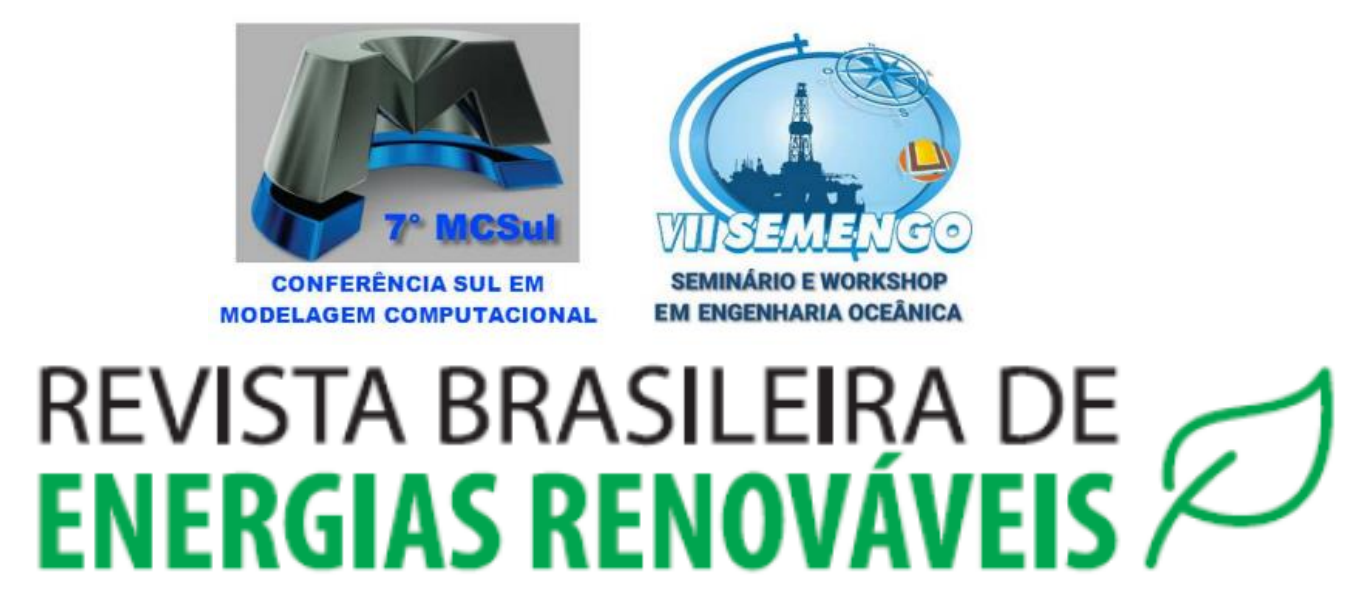

\title{
ESTUDO NUMÉRICO DO ESCOAMENTO TURBULENTO EM CHAMINÉ SOLAR PARA VENTILAÇÃO DE AMBIENTES CONSTRUIDOS ${ }^{1}$
}

\author{
RODRIGO SPOTORNO VIEIRA ${ }^{2}$, MURIEL LABORDE RAJÃO ${ }^{2}$, LIÉRCIO ANDRÉ \\ ISOLDI $^{2}$, LUIZ ALBERTO OLIVEIRA ROCHA $^{3}$, ELIZALDO DOMINGUES DOS \\ SANTOS $^{2}$
}

\begin{abstract}
${ }^{1}$ Apresentado no $7^{\circ}$ Conferência Sul em Modelagem Computacional (MCSul) e do VII Seminário e Workshop em Engenharia Oceânica (SEMENGO).

${ }^{1}$ Universidade Federal do Rio Grande, Escola de Engenharia Avenida Itália, km 8, Rio Grande, RS, Brasil. spotorno.furg@gmail.com, liercioisoldi@furg.br, brasil.murilaborde@yahoo.com.br, elizaldosantos@furg.br

${ }^{2}$ Universidade Federal do Rio Grande do Sul Programa de Pós-Graduação em Engenharia Mecânica Rua Sarmento Leite, 425, Porto Alegre, RS, Brasil, luizrocha@mecanica.ufrgs.br
\end{abstract}

\section{RESUMO}

O presente trabalho tem como objetivos propor uma metodologia numérica para predizer o comportamento de escoamentos turbulentos em ambientes conectados a uma chaminé solar e avaliar a influência da geometria da chaminé sobre o desempenho do escoamento no ambiente através do método Design Construtal. O sistema consiste em um escoamento bidimensional, incompressível, turbulento e com transferência de calor por convecção natural em um ambiente representado por uma região retangular conectado a uma chaminé solar com parede passiva inclinada (apenas transmite o fluxo radiante incidente). A simulações foram realizadas com Rayleigh $\mathrm{Ra}_{\mathrm{H}}=1,36 \times 10^{14}$ e número de Prandtl de aproximadamente $\operatorname{Pr}=0,7$. No 
estudo de avaliação geométrica busca-se obter a melhor vazão mássica de ar no domínio computacional. O sistema é submetido a duas restrições, dadas pela área da chaminé e a área do ambiente construído. A geometria da chaminé (que foi avaliada no presente trabalho) é submetida a dois graus de liberdade: $W_{\mathrm{e}} / W_{\mathrm{g}}$ e $W_{\mathrm{e}} / H_{\mathrm{t}}$. Sendo $W_{e}$ a base superior da chaminé, saída, $W_{g}$ base inferior da chaminé e $H_{t}$ sua altura total. Para todos os casos foi considerada uma relação $W_{\mathrm{e}} / H_{t}=0,1$. As equações temporais médias de conservação de massa, quantidade de movimento e energia são resolvidas através do método dos volumes finitos (MFV). Para fechamento das equações médias no tempo é empregado o modelo a duas equações $k-\varepsilon$. A vazão mássica obtida com o presente modelo foi concordante dentro de $92,3 \%$ com os resultados propostos na literatura, inclusive para o fator adimensional avaliado na literatura estando dentro de 99,7\% de concordância, para um caso específico (cuja inclinação da parede passiva foi de $4^{\circ}$ ). Com relação a avaliação geométrica, foi observado que para a faixa de geometrias estudadas $\left(0.1 \leq W_{\mathrm{e}} / W_{\mathrm{g}} \leq 1.0\right)$ a vazão mássica máxima foi obtida no extremo superior, ou seja, $\left(W_{\mathrm{e}} / W_{\mathrm{g}}\right)_{\mathrm{o}}=1.0$. Além disso, foi observada uma diferença na vazão mássica de 71,6\% entre a geometria ótima e a pior geometria, mostrando a aplicabilidade do Design Construtal neste problema.

Palavras chave: chaminé solar, ventilação, vazão mássica, avaliação geométrica, Design Construtal.

\title{
NUMERICAL STUDY OF TURBULENT FLOW WITH NATURAL CONVECTION FOR VENTILATION OF CONSTRUCTED ENVIRONMENTS
}

\begin{abstract}
The present work aims to propose a numerical methodology to predict the behavior of turbulent flows in environments connected to a solar chimney and to evaluate the influence of the chimney geometry on the flow performance in the environment through the Design Construtal method. The system consists of a two-dimensional, incompressible, turbulent flow with heat transfer by natural convection in an environment represented by a rectangular region connected to a solar chimney with a sloping passive wall (it only transmits the incident radiant flux). Simulations were performed with Rayleigh $\mathrm{Ra}_{H}=1.36 \times 10^{14}$ and Prandtl number of approximately $\operatorname{Pr}=0.7$. In the geometric evaluation study, it is sought to obtain the best mass flow rate of air in the computational domain. The system is subjected to two restrictions, given by the chimney area and the built environment area. The chimney geometry
\end{abstract}


(which was evaluated in the present work) is submitted to two degrees of freedom: $W_{\mathrm{e}} / W_{\mathrm{g}}$ and $W_{\mathrm{e}} / H_{\mathrm{t}}$. Being $W_{\mathrm{e}}$ the upper base of the chimney, outlet, $W_{g}$ bottom base of the chimney and $H_{t}$ its total height. For all cases a $W_{\mathrm{e}} / H_{\mathrm{t}}=0.1$ ratio was considered. The mean temporal equations of conservation of mass, momentum and energy are solved by the finite volume method (MFV). To close the mean time equations, the model is applied to two equations $k-\varepsilon$. The mass flow rate obtained with the present model was concordant within $92.3 \%$ with the results proposed in the literature, including for the dimensionless factor evaluated in the literature being within $99.7 \%$ agreement, for a specific case (whose slope of the passive wall was $4^{\circ}$ ). With respect to the geometric evaluation, it was observed that for the studied geometry range $\left(0.1 \leq W_{\mathrm{e}} / W_{\mathrm{g}} \leq 1.0\right)$ the maximum mass flow rate was obtained at the upper end, i.e. $\left(W_{\mathrm{e}} / W_{\mathrm{g}}\right)_{\mathrm{o}}$ $=1.0$. In addition, a difference in mass flow of $71.6 \%$ was observed between the optimum geometry and the worst geometry, showing the applicability of the Construtal Design in this problem.

Keywords: solar chimney, ventilation, mass flow, geometric evaluation, Design Construtal.

\section{INTRODUÇÃO}

A possibilidade do uso de ferramentas numéricas tanto para nível científico quanto empresarial, visando à viabilização de projetos, contribui para uma aceleração de processos tanto de produtos quanto para obtenção de recomendações teóricas para os problemas de engenharia, uma vez que o quadro atual necessita de processos teóricos que auxiliem o processo experimental. Atualmente a busca por energia limpa a realização de experimentos teóricos agrega aos procedimentos da engenharia. O Brasil enfrentou diversas crises energéticas, o que motivou o aumento dos estudos por busca da geração de energia de formas alternativas, embora esse pensamento seja de uma forma global, a busca por geração de energia elétrica de forma limpa no Brasil é uma prática de extrema importância, visto que somos ricos em recursos naturais, afirmava a publicação de 2010 no Portal Brasil "O Brasil possui a matriz energética mais renovável do mundo industrializado com 45,3\% de sua produção proveniente de fontes como recursos hídricos, biomassa e etanol, além das energias eólica e solar." O trabalho que será apresentado parte de estudos anteriores, buscando-se a melhor maneira de aumentar a vazão mássica de ar em um ambiente conectado a uma espécie de chaminé solar. Um importante estudo foi desenvolvido por Khanal e Lei (2015) que relataram uma investigação numérica do escoamento turbulento com convecção natural. Neste estudo foi apresentado uma chaminé solar de parede passiva inclinada (IPWSC - do inglês: 
inclined passive wall solar chimney). Ela consiste basicamente de uma chaminé, anexa a um ambiente da casa, com uma parede de vidro inclinada e um absorvedor, que capta e transforma a energia solar em energia térmica. As paredes foram consideradas como as fronteiras térmicas e por isso foi modelado considerando, a altura da parede do absorvedor $\left(H_{\mathrm{a}}\right)$, a espessura da parede do absorvedor $\left(W_{\mathrm{t}}\right)$, o tamanho da janela - entrada $\left(W_{\mathrm{I}}\right)$, o tamanho da abertura de entrada para chaminé $\left(H_{\mathrm{I}}\right)$, a altura $(H)$ e comprimento $(L)$ do espaço ventilado (quarto) também são mantidos constantes, desconsiderando a perda de calor através do vidro, conforme mostra a Fig. 1. O escoamento de ar se movimenta a partir da diferença de temperatura no domínio que causa uma variação de massa especifica, gerando uma força de campo que movimenta o fluido. A relação matemática $A=H_{d} / W_{\mathrm{g}}$ foi a usada por Khanal e Lei (2015) para apresentar a sua restrição do problema.

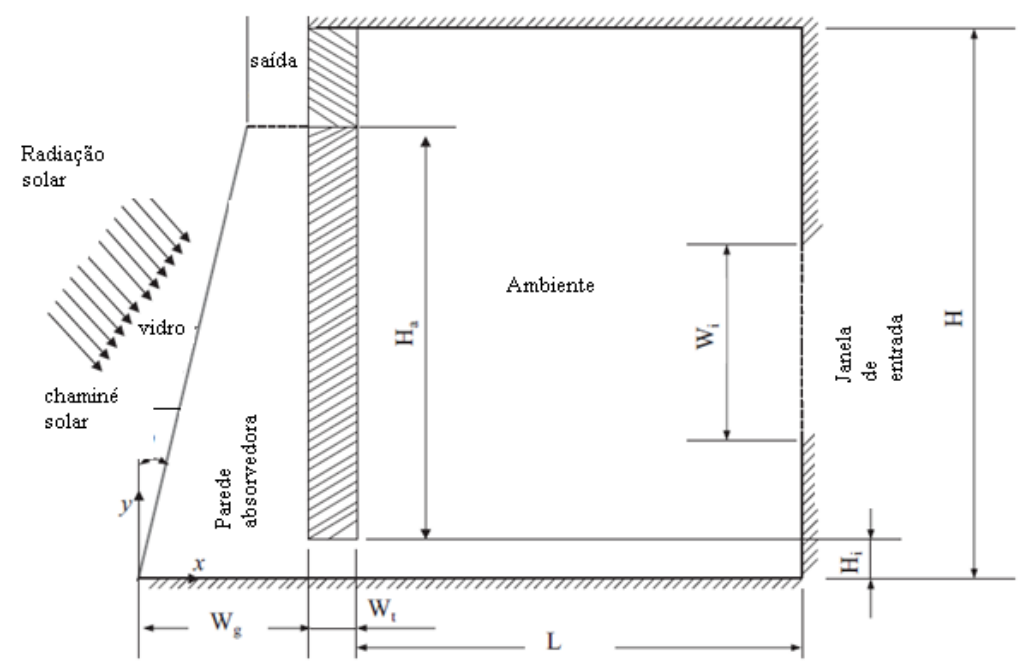

Figura 1- Esquema do princípio de funcionamento da chaminé solar de parede inclinada acoplado a um ambiente à ser ventilado (Adaptado de Khanal e Lei, 2015)

Apesar de ser uma era com um crescente aumento do uso de condicionadores de ar nas residências é necessário pensar em formas alternativas para busca de um ambiente ameno. $\mathrm{O}$ uso dos climatizadores para condicionamento do ar é bastante eficiente, entretanto geram consumo de energia elétrica. Um aparelho de 12000 BTU consome cerca de 33,6 KWh tendo um custo diário $\mathrm{R} \$ 16,23$ se operar durante 24 horas, segundo simulador da página da CCEE. Buscar possibilidades que não demandem esse consumo pode tornar-se de grande atrativo para os usuários. Analisando também por outro viés, é possível citar com muita propriedade a necessidade de ambientes ventilados. Cada vez mais percebemos pessoas com problemas respiratórios que necessitam ficar em lugares onde exista maior renovação de ar. Embora os 
sistemas de ar condicionados possam ser projetados para ter um número de renovações de ar, a ideia é aproveitar a energia solar para auxiliar esse mecanismo. Pensando nisso está sendo proposto um estudo na qual a chaminé solar possa atuar conectada a um ambiente fazendo renovar o volume de ar dos interiores, por diferença de massa especifica fazer o ar circular no ambiente.

Existem diversos estudos desenvolvidos sobre chaminé solar, onde se busca otimizar a geometria da mesma visando aumentar a vazão mássica no seu interior. Uma vez que esse aumento gera benefícios que podem ser usados como forma de força motriz em uma turbina ou utilizados como meio para ventilação de interiores na busca de conforto térmico. Bassiouny e Korah (2008) analisou o efeito do ângulo de inclinação de uma chaminé solar e concluiu que a melhor disposição se encontra entre $45^{\circ}$ e $70^{\circ}$. Importante ressaltar que esse trabalho, a disposição da chaminé, é diferente do estudo de Khanal e Lei (2015), que por sua vez estudava a inclinação da parede de vidro. No estudo de Zamora e Kaiser (2010) o principal objetivo foi analisar os efeitos dos parâmetros relevantes do comportamento dinâmico e térmico dentro da chaminé solar. Cavalcante (2010) realizou uma investigação em oito cidades do país, com zonas bioclimáticas diferentes, para verificar se o dispositivo da chaminé anexa ao um ambiente apresenta resultados satisfatórios para o território brasileiro. Imran et al. (2015) realizaram o estudo de simulação numérica analisando o desempenho interno da chaminé solar, propondo variação da geometria da mesma para condições ambientais no Iraque. As últimas duas simulações, em território brasileiro e Iraquiano utilizaram disposição física da chaminé semelhante a que Bassiouny e Korah (2008) investigaram.

Vieira (2015) em sua proposta de pós-graduação em Engenharia Oceânica apresentou um estudo da influência geométrica de uma chaminé solar, aplicando o método do Designer Construtal e resolvendo o problema numericamente através de simulação numérica, com software de volumes finitos FLUENT. O seu objetivo foi a investigação em uma Usina de Chaminé Solar bem como a influência de alguns parâmetros geométricos sobre a potência do dispositivo buscando otimização, é uma outra aplicação da chaminé solar (para conversão da energia solar em energia elétrica). A Usina de Chaminé Solar vem sendo estudada e basicamente se trata de uma proposta de planta de energia renovável que transforma energia solar em eletricidade. Composta de o coletor, a chaminé e as turbinas. Baseia-se nos princípios efeito estufa, empuxo do efeito chaminé e energia eólica.

Diante disso, tomamos como problema de pesquisa comprovar o modelo utilizado verificando-o com o estudo que Khanal e Lei (2015) realizaram. E buscar uma nova 
geometria que permita uma eficiência de vazão mássica de ar semelhante ao trabalho de Khanal e Lei (2015). Mantendo a área da chaminé constante visando ser o parâmetro restritivo, e variando apenas um grau de liberdade $W_{e} / W_{g}$, ou seja o outro grau de liberdade $W_{e} / H_{t}$ foi mantido constate. Para isso temos como metodologia para produção desse artigo a pesquisa bibliográfica. As equações de conservação de massa, quantidade de movimento e energia médias no tempo bem como as equações de transporte do modelo de turbulência $\mathrm{k}-\varepsilon$ são resolvidas numericamente através do método de volumes finitos (Patankar, 1980; Versteeg e Malalasekera, 2007). Mais precisamente é empregado o software de Dinâmica dos Fluidos Computacional (CFD) FLUENT (FLUENT, 2007). Para realizar a segunda etapa do trabalho utilizou - se a razão $A=W_{e} / W_{g}$ para apresentar a restrição do problema, ao presente trabalho quando a investigação for a nova geometria.

\section{MATERIAL E MÉTODOS}

Um problema desta magnitude pode ser resolvido através das equações da massa, quantidade de movimento e energia, porém uma modelagem numérica possibilita a solução. Primeiramente foi realizado a simulação para comprovar o modelo utilizado, que foi verificado com os valores encontrados por Khanal e Lei (2015). Dentre as possibilidades de simulação foi testado para um regime permanente e regime transiente. O objetivo tanto da publicação mencionada como o deste trabalho é maximizar a vazão mássica que passa pela saída da chaminé. Sendo a vazão mássica dada por a multiplicação da massa especifica $\left(\mathrm{kg} / \mathrm{m}^{3}\right)$, da abertura de saída da chaminé e da média das velocidades médias na saída da chaminé, conforme a seguinte expressão:

$$
\dot{m}=\rho w e \overline{v e}
$$

Khanal e Lei (2015) propuseram um valor de vazão mássica adimensional a título apena comparativo. Em virtude da avaliação desenvolvida sobre o trabalho publicado, a mesma variável foi resolvida aqui para efeitos de verificação. A vazão mássica adimensional é obtida pela razão entre a vazão mássica por unidade de comprimento (por se tratar de um problema bidimensional) pela massa especifica vezes a difusividade térmica. 


$$
\dot{M}=\dot{m} / \rho \alpha
$$

\section{Descrição do domínio computacional e condições de contorno}

As condições de contorno são as mesmas estabelecidas por Khanal e Lei (2015), ou seja, a parede HI é submetida a um fluxo de calor imposto imitando o efeito do absorvedor no dispositivo, nas superfícies CD e JI impõe-se uma pressão atmosférica. As demais superfícies possuem condições de não deslizamento, impermeabilidade e isolamento térmico. Para chaminé avaliada todas as dimensões serão constantes exceto a parede AJ e IJ que variam de acordo com o angulo de inclinação da parede inclinada. Então para chaminé solar de parede inclinada com anexo foi adotado as paredes verticais BC, DE, FG e HI, as paredes horizontais $\mathrm{AB}, \mathrm{EF}$ e GH e a parede inclinada JA. O fluxo de calor considerado constante e as paredes, exceto a do absorvedor, assumiram perfil adiabático, conforme Fig. 2.

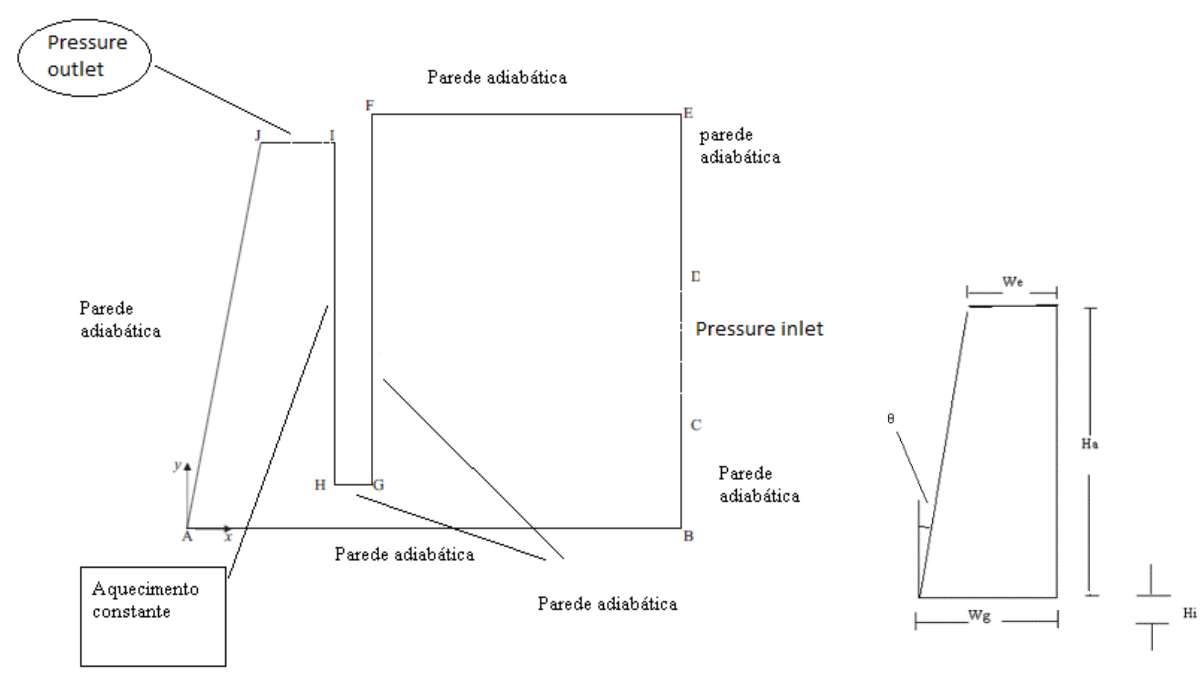

Figura 2- Esquema da condição de contorno do domínio computacional (Adaptado de Khanal e Lei, 2015).

O estudo teve como objetivo buscar uma forma geométrica que permitisse a maximização da vazão mássica. Contudo, em Khanal e Lei (2015) a área da abertura da chaminé não foi mantida constate. Sendo que o ângulo da parede de vidro e o comprimento da abertura de saída da chaminé seriam as únicas variáveis. As demais dimensões permaneceram constantes. $\mathrm{O}$ ângulo investigado variou de $0^{\circ}$ a $6^{\circ}$. A parede de vidro era articulada o que possibilitava a variação desse ângulo da parede de vidro com a vertical. Simulações realizadas 
possuem uma maior validade para aquecimento solar realizada com emissão de energia constante. A Tabela 1 apresenta os principais dados empregados no caso de verificação.

Tabela 1. Parâmetros físicos e numéricos empregados na verificação.

\begin{tabular}{c|c|c|c|c|c|c}
\hline Fluido & $\begin{array}{c}\text { Número } \\
\text { de Prandtl }\end{array}$ & Rayleigh & Modelo de turbulência & $\begin{array}{c}\text { Fluxo de } \\
\text { calor }\end{array}$ & $\begin{array}{c}\text { Ângulo de } \\
\text { inclinação da } \\
\text { parede passiva }\end{array}$ & Regime \\
\hline $\mathrm{Ar}$ & 0,7 & $1,36 \times 10^{14}$ & $\mathrm{k}-\varepsilon$ padrão & $1000 \mathrm{~W} / \mathrm{m}^{2}$ & $4^{\circ}$ & Transiente \\
\hline
\end{tabular}

A pressão de saída (ambiente), variação das componentes de velocidade, temperatura, energia cinética turbulenta e taxa de dissipação são desprezíveis. A Tabela 2 abaixo resume as condições na entrada e saída.

As simulações são realizadas com os parâmetros geométricos, listados na tabela 3, determinados por Khanal e Lei (2015). Tanto o trabalho de verificação da publicação de Khanal e Lei quanto o trabalho visando buscar uma nova geometria que otimize a vazão mássica foram baseados nos parâmetros abaixo.

Tabela 2 Condições de entrada e saída

\begin{tabular}{|c|c|c|c|c|}
\hline \multirow{4}{*}{ Entrada } & \multicolumn{4}{|c|}{ Entrada do ambiente trecho CD Janela } \\
\hline & Temperatura & To & $300 \mathrm{~K}$ & $27^{\circ} \mathrm{C}$ \\
\hline & Intensidade turbulenta & & $3 \%$ & \\
\hline & Pressão manométrica & & 0 & \\
\hline \multirow{4}{*}{ Saída } & \multicolumn{4}{|c|}{ Sáida da chaminé trecho JI Boca da chaminé } \\
\hline & Temperatura & To & $300 \mathrm{~K}$ & $27^{\circ} \mathrm{C}$ \\
\hline & Intensidade turbulenta & & $3 \%$ & \\
\hline & Pressão manométrica & & 0 & \\
\hline
\end{tabular}


Tabela 3 Parâmetros geométricos

\begin{tabular}{c|c}
\hline \multicolumn{2}{c}{ Parâmetros geométricos } \\
\hline$H_{\mathrm{a}} / W_{\mathrm{G}}$ & 6,25 \\
\hline$H / H_{\mathrm{a}}$ & 1,2 \\
\hline$H_{\mathrm{a}} / W_{\mathrm{i}}$ & 2,5 \\
\hline$L / W_{\mathrm{t}}$ & 12,5 \\
\hline$H_{\mathrm{a}}$ & $2,5 \mathrm{~m}$ \\
\hline
\end{tabular}

\section{Modelagem matemática empregada no software FLUENT ${ }^{\circledR}$}

O trabalho visa maximizar a vazão mássica de fluido, ar, que escoa em determinado ambiente, então quando se busca uma solução do escoamento incompressível, turbulento, em um domínio bidimensional com transferência de calor por convecção natural é necessário resolver as equações de conservação de massa, quantidade de movimento em $x$ e $y$ e energia médias no tempo são dadas, respectivamente, por (Bejan, 2004). As propriedades termo físicas mantidas constantes, exceto a massa especifica, uma vez que pela aproximação de Boussinesq o efeito da massa especifica é variável sobre o empuxo pois essa variação é que induz o movimento do fluido. A referência $0^{\circ}$ da parede passiva é na configuração vertical. Condições adotadas para entrada do ambiente e condições adotadas para saída da chaminé, pressão manométrica, temperatura de referência e intensidade turbulenta. Sendo a intensidade turbulenta usada 3\%, conforme o trabalho de Khanal e Lei (2015). Importante ressaltar que a intensidade turbulenta depende da espessura da camada limite.

Conservação da massa:

$$
\frac{\partial \bar{u}}{\partial x}+\frac{\partial \bar{v}}{\partial y}=0
$$

Conservação da quantidade de movimento 
Em $x$

$$
\frac{\partial \bar{u}}{\partial t}+\left(\bar{u} \frac{\partial \bar{u}}{\partial x}+\bar{v} \frac{\partial \bar{u}}{\partial y}\right)=-\frac{1}{\rho} \frac{\partial \bar{P}}{\partial x}+\left(v+v_{t}\right)\left(\frac{\partial^{2} \bar{u}}{\partial x^{2}}+\frac{\partial^{2} \bar{u}}{\partial y^{2}}\right)
$$

$\operatorname{Em} y$

$$
\frac{\partial \bar{v}}{\partial t}+\left(-\frac{\partial \bar{v}}{\partial x}+\bar{v} \frac{\partial \bar{v}}{\partial y}\right)=-\frac{1}{\rho} \frac{\partial \bar{P}}{\partial y}+\left(v+v_{t}\right)\left(\frac{\partial^{2} \bar{v}}{\partial x^{2}}+\frac{\partial^{2} \bar{v}}{\partial y^{2}}\right)+g \beta\left(\bar{T}-T_{0}\right)
$$

Energia:

$$
\frac{\partial \bar{T}}{\partial t}+\left(\bar{u} \frac{\partial \bar{T}}{\partial x}+\bar{v} \frac{\partial \bar{T}}{\partial y}\right)=\left(\alpha+\alpha_{t}\right)\left(\frac{\partial^{2} \bar{T}}{\partial x^{2}}+\frac{\partial^{2} \bar{T}}{\partial y^{2}}\right)
$$

Equação para transporte de energia cinética turbulenta $k$ e taxa de dissipação de energia turbulenta $(\varepsilon)$ :

$$
\begin{aligned}
& \frac{\partial k}{\partial t}+\frac{\partial(u k)}{\partial x}+\frac{\partial(v k)}{\partial y}=\frac{1}{\rho} \frac{\partial}{\partial x}\left[\left(\mu+\frac{\mu t}{\sigma k}\right) \frac{\partial k}{\partial x}\right]+\frac{1}{\rho} \frac{\partial}{\partial y}\left[\left(\mu+\frac{\mu t}{\sigma k}\right) \frac{\partial k}{\partial y}\right]+\frac{1}{\rho}\left[G_{k}+G_{b}\right]-\varepsilon \\
& \frac{\partial \varepsilon}{\partial t}+\frac{\partial(u s)}{\partial x}+\frac{\partial(v \varepsilon)}{\partial y}=\frac{1}{\rho} \frac{\partial}{\partial x}\left[\left(\mu+\frac{\mu t}{\sigma \varepsilon}\right) \frac{\partial s}{\partial x}\right]+\frac{1}{\rho} \frac{\partial}{\partial y}\left[\left(\mu+\frac{\mu t}{\sigma s}\right) \frac{\partial s}{\partial y}\right]+C_{1} \varepsilon \frac{\varepsilon}{\rho k}\left(G_{k}+C_{3} \varepsilon G_{b}\right)- \\
& C_{2} \varepsilon \frac{\varepsilon^{2}}{k}
\end{aligned}
$$

onde $G_{k}$ e $G_{b}$ são calculados por:

$$
\begin{aligned}
& G_{b}=\beta g i \frac{\mu t}{\sigma t} \frac{\partial(\bar{T})}{\partial x i} \\
& G_{k}=\mu t\left(\frac{\partial \overline{m i}}{\partial x j}+\frac{\partial \overline{m j}}{\partial x j}\right)\left(\frac{\partial \overline{m i}}{\partial x j}\right)-\frac{2}{3} \rho k \delta_{i j} \frac{\partial \overline{m i}}{\partial x j}
\end{aligned}
$$


onde ( ${ }^{-}$) representa o operador de média temporal; $\rho$ é a massa específica do fluido $\left(\mathrm{kg} / \mathrm{m}^{3}\right) ; \mu$ é a viscosidade dinâmica $(\mathrm{kg} / \mathrm{ms}) ; \mu_{t}$ é a viscosidade turbulenta $(\mathrm{kg} / \mathrm{ms})$; $v$ é a viscosidade cinemática $\left(\mathrm{m}^{2} / \mathrm{s}\right) ; \alpha$ é a difusividade térmica $\left(\mathrm{m}^{2} / \mathrm{s}\right)$ dada por $\mathrm{k} / \rho C_{\mathrm{p}} ; \alpha_{t}$ é a difusividade térmica turbulenta $\left(\mathrm{m}^{2} / \mathrm{s}\right)$; $\bar{u}$ é a velocidade média na direção $x, ; \bar{v}$ é a velocidade média na direção $y ; P$ é a pressão $(\mathrm{N} / \mathrm{m}) ; T$ é a temperatura $(\mathrm{K}) ; g_{i}$ é a aceleração da gravidade na direção $y ; t$ representa o domínio do tempo (s); $\varepsilon$ taxa de dissipação de energia cinética turbulenta; $k$ energia cinética turbulenta $\left(\mathrm{m}^{2} / \mathrm{s}^{2}\right) ; \beta$ coeficiente de expansão térmica e $k$ condutividade térmica do ar; $\delta_{i j}$ é o delta de Kronecker; $\operatorname{Pr}$ número de Prandtl dado por v/ $\alpha$.

\section{Modelagem numérica empregada no software FLUENT ${ }^{\circledR}$}

Khanal e Lei (2015) realizaram simulações com duas malhas 25.650 e 42.510 elementos. A malha mais grosseira foi a escolhida uma vez que houve uma diferença de $2 \%$ na vazão mássica entre a mais grosseira e a mais refinada. Para verificação do modelo, serão mantidas as condições impostas no trabalho de Khanal e Lei (2015), uma chaminé solar de parede passiva com uma inclinação de $4^{\circ}$ com a vertical e com ambiente anexo. Posteriormente, serão testadas novas geometrias, através da aplicação do Design Construtal, com o objetivo de maximizar a vazão mássica do dispositivo. O estudo terá dois graus de liberdade, porém um será mantido constante $W_{e} / H_{t}$. variando apenas $W_{e} / W_{g}$ de 0,1 a 1 . Tanto para a primeira parte do trabalho, que é a verificação do trabalho de Khanal e Lei (2015) quanto para investigação da melhor geometria foram utilizadas malhas com 37400 elementos quadrados. A equação de área da chaminé é a dada pela área a de um trapézio, que é a restrição do problema e dada por:

$$
A t=\frac{(W \theta+W g)}{2} *(H t)
$$

Entretanto como há uma equações e três variáveis é necessário a introdução de dois graus de liberdade para o fechamento do problema. Neste caso os graus de liberdade são $W_{e} / H_{t}$. que foi considerado constante e igual a 0,1 e $W_{e} / W_{g}$ que variou de 0,1 a 1 . Valor da área constante e igual a $0,944625 \mathrm{~m}^{2}$. Vale destacar que no absorvedor foi empregado um fluxo de calor constante de $1000 \mathrm{~W} / \mathrm{m}^{2}$ como condição de contorno. Com relação a solução transiente, foram empregados 100 números de passo de tempo com $\Delta t=0,01$ s e 300 iterações para convergência de cada intervalo de passo de tempo. Para o acoplamento pressão 
velocidade foi empregado o algoritmo SIMPLE. As simulações foram consideradas convergidas quando os resíduos para massa, velocidades, energia e para as equações do modelo de turbulência (transporte de $k$ e $\varepsilon$ ) entre duas iterações consecutivas foram menores do que $10^{-6}, 10^{-6}, 10^{-8}$ e $10^{-6}$, respectivamente.

\section{RESULTADOS E DISCUSSÃO}

Para mostrar os resultados das simulações encontrados, um escoamento com $\mathrm{R}_{\mathrm{aH}}=1.36 \times 10^{14}$ e $\mathrm{P}_{\mathrm{r}}=0.71$ foi simulado com o presente modelo. Na simulação foi realizada uma tentativa de obter os campos de velocidades e intensidade de turbulência a partir de um modelo no regime permanente, ou seja, sem a solução dos termos transientes nas equações de conservação de quantidade de movimento e energia. As Figuras 4 e 5 ilustram, respectivamente, a intensidade de turbulência e o campo de velocidades para o escoamento no regime permanente (Figs. 4(a) e 5(a)) e transiente (Figs. 4(b) e 5(b)) obtidos no presente trabalho. Em uma comparação qualitativa da solução permanente com os resultados de Khanal e Lei (2015) é possível afirmar que os campos obtidos ainda estão em desenvolvimento, apesar de em alguns pontos (como na região de saída da chaminé) os mesmos terem atingido soluções próximas as propostas na literatura. Esse comportamento, provavelmente ocorre devido a baixa convergência obtida para os resíduos na solução numérica. Em função do comportamento obtido com as soluções no regime permanente, testou-se um novo modelo levando em consideração os termos transientes das equações de conservação de massa, quantidade de movimento e energia médias no tempo com o objetivo de reduzir a magnitude dos resíduos de convergência do modelo numérico. As Figuras 4b e 5b ilustram, respectivamente, o campo de intensidade de turbulência e velocidade para o regime transiente. O comportamento qualitativo dos campos fluidodinâmico e térmico foram próximos aos preditos por Khanal e Lei (2015). Um exemplo pode ser visto na Fig. 6, onde o campo de intensidade de turbulência encontrado na literatura para uma parede de vidro com

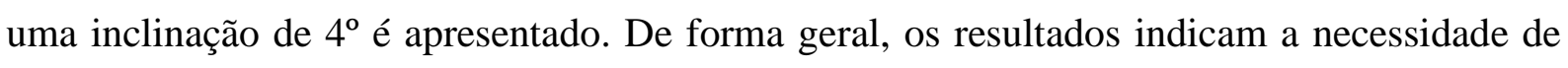
uma solução transiente para este problema.

O perfil de intensidade de turbulência encontrado por Khanal e Lei (2015) para a chaminé solar com parede de vidro com um ângulo de inclinação de $4^{\circ}$ está representado na figura 6.

A Tabela 4 demonstra os resultados encontrados no presente trabalho e os obtidos por Khanal e Lei (2015). Mais precisamente, foram comparadas a vazão mássica 
adimensional, a vazão mássica e a magnitude média do perfil de velocidades médias na saída da chaminé. É possível perceber que as vazões mássicas são muito próximas com uma diferença de $0,0111 \mathrm{~kg} / \mathrm{s}$, ou seja, o resultado encontrado foi $92,3 \%$ concordante com o resultado da literatura. E que em relação ao termo adimensional que Khanal e Lei (2015) sugeriu, foi encontrado um desvio em termos percentuais de aproximadamente $0,3 \%$, ou seja, $99,3 \%$ dos valores forma concordantes.
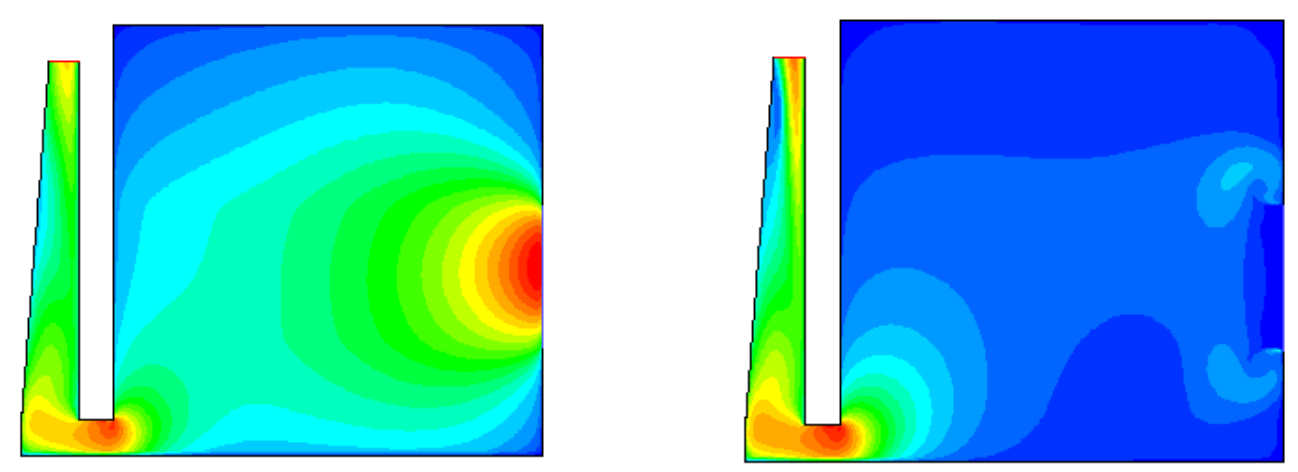

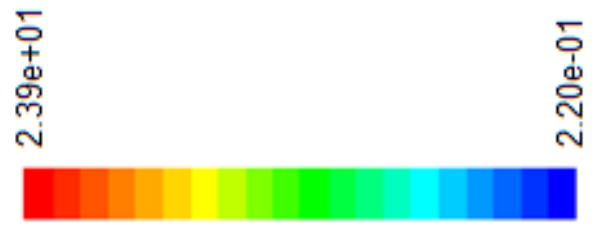

a)

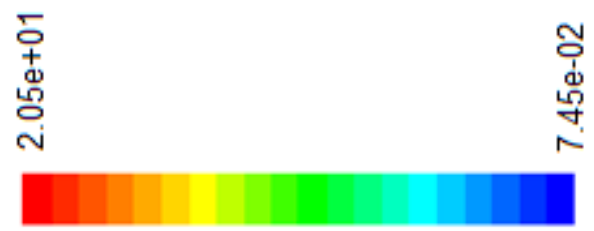

b)

Figura 3 - Campo de intensidade de turbulência para um escoamento a $\mathrm{Ra}_{\mathrm{H}}=1,36 \times 10^{14}$ e $\mathrm{Pr}$ $=0,71$ : a) solução no regime permanente, b) campo permanente obtido com solução transiente. 
Tabela 4- Comparativo de resultados entre o presente trabalho e os obtidos por Khanal e Lei (2015).

\begin{tabular}{ccccc}
\hline & $\mathrm{Ra}$ & $\dot{M}$ & $\dot{m}(\mathrm{Kg} / \mathrm{s})$ & $\overline{v_{e}}(\mathrm{~m} / \mathrm{s})$ \\
\hline Khanal e Lei (2015) & $1,36 \times 10^{14}$ & 5100,0 & 0,1439 & 0,55 \\
\hline Presente trabalho & $1,36 \times 10^{14}$ & 5084,8 & 0,1328 & 0,56 \\
\hline
\end{tabular}
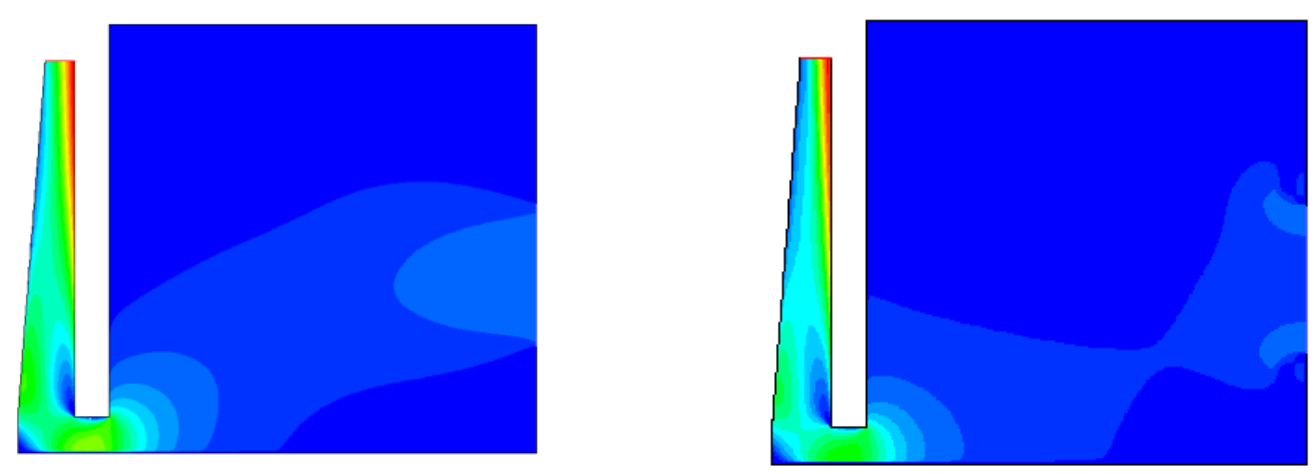

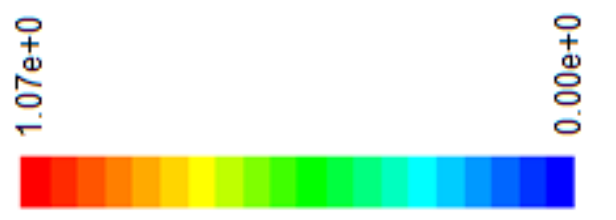

a)

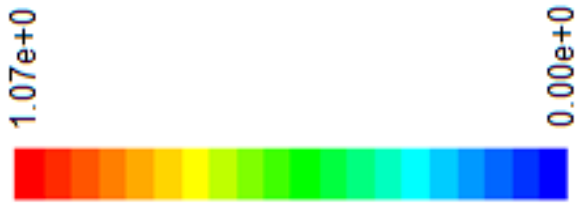

b)

Figura 4 - Campo de velocidades médio para um escoamento a $\mathrm{RaH}_{\mathrm{H}}=1,36 \times 10^{14}$ e $\operatorname{Pr}=0,71$ : a) solução no regime permanente, b) campo permanente obtido com solução transiente. 


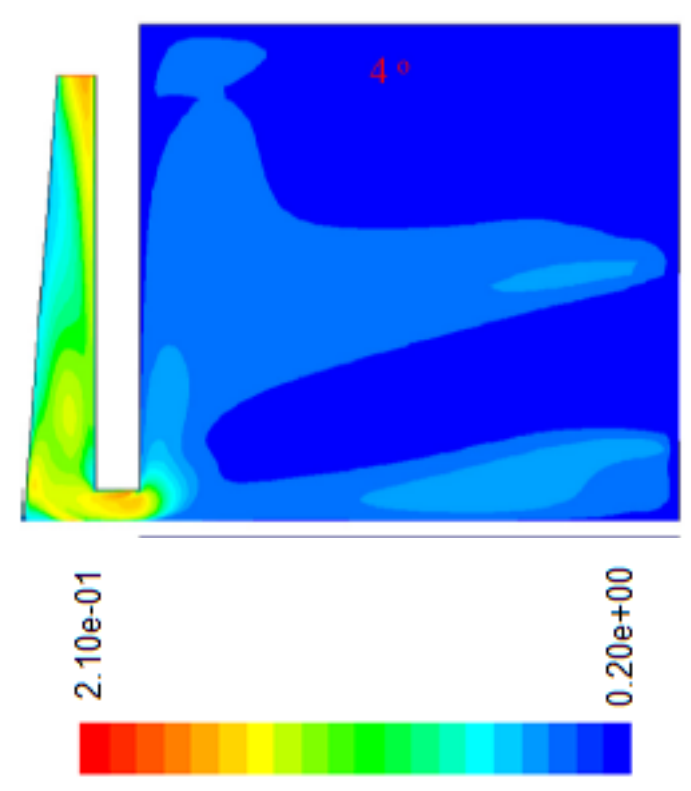

Figura 5 - Intensidade de turbulência para chaminé com inclinação de $4^{\circ}$ para a parede de vidro para uma solução de escoamento no regime transiente obtido na literatura (Fonte Khanal e Lei (2015)).

A Figura 7 apresenta o perfil de velocidades média na saída da chaminé. Pode ser observado que a velocidade aumenta quanto mais próxima vai ficando do absorvedor, passando de um metro por segundo, entretanto em virtude da parede ela tende a zero novamente.

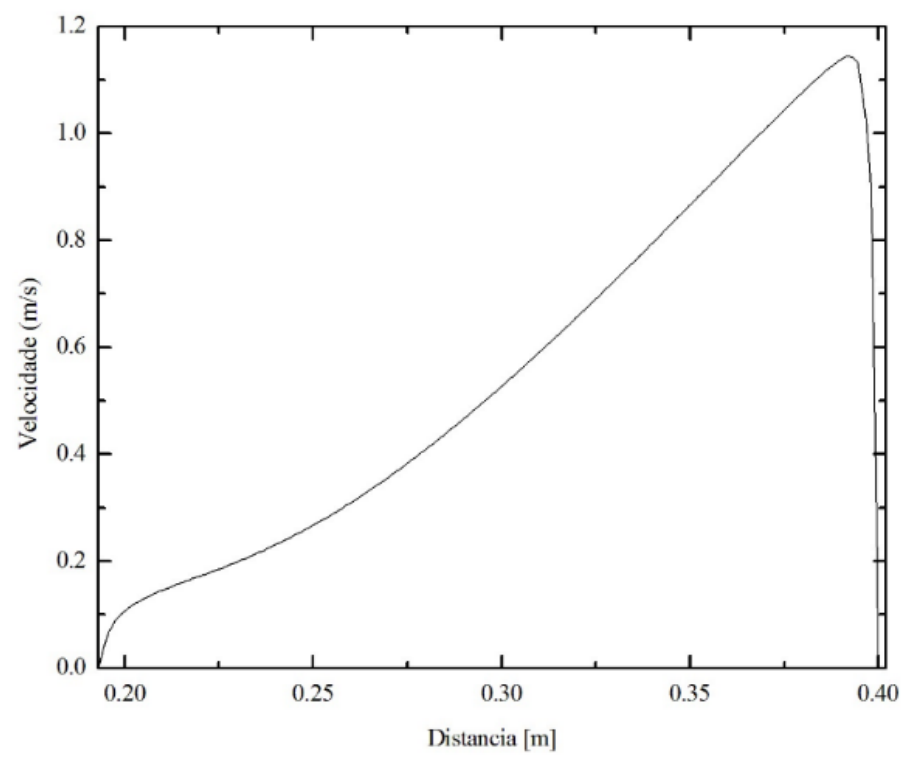

Figura 6 - Perfil médio de velocidade na saída da chaminé quando o escoamento atinge o regime permanente. 


\section{Avaliação Geométrica com Design Construtal}

A segunda parte deste trabalho consiste em realizar simulações buscando a melhor vazão mássica de ar, mantendo a área da chaminé constante e avaliando o efeito de um grau de liberdade sobre o desempenho do sistema. Mais precisamente, manteve-se uma área para a chaminé de $\mathrm{A}=0,94 \mathrm{~m}^{2}$, que foi definida com base no melhor caso estudado em Khanal e Lei (2015), a razão $W_{\mathrm{e}} / W_{\mathrm{g}}$ foi variada na faixa $0,1 \leq W_{\mathrm{e}} / W_{\mathrm{g}} \leq 1,0$ e a razão $W_{\mathrm{e}} / H_{\mathrm{t}}$ foi mantida constante com $W_{\mathrm{e}} / H_{\mathrm{t}}=0,1$. A Figura 8 apresenta o efeito da razão $W_{\mathrm{e}} / W_{\mathrm{g}}$ sobre a vazão mássica que escoa através da seção de saída da chaminé. Pode ser observado que, para as configurações geométricas avaliadas, houve um aumento da vazão mássica com o aumento da razão $W_{\mathrm{e}} / W_{\mathrm{g}}$, obtendo-se uma vazão uma vez maximizada de $m_{\mathrm{m}}=0,28 \mathrm{~kg} / \mathrm{s}$ para a razão ótima $\left(W_{\mathrm{e}} / W_{\mathrm{g}}\right)_{\mathrm{o}}=1,0$. Além disso, foi observada uma diferença na vazão mássica de 71,6\% entre a geometria ótima e a pior geometria, mostrando a aplicabilidade do Design Construtal neste problema.

No que tange a velocidade na saída da chaminé pode se constatar através da Figura 9, onde é apresentado o efeito da razão $W_{e} / W_{g}$ sobre a magnitude média de velocidades na saída da chaminé, que obteve-se a maior magnitude do campo de velocidades para a relação de $W_{\mathrm{e}} / W_{\mathrm{g}}=0,7$ com um pico muito próximo de $v_{\mathrm{e}}=0,65 \mathrm{~m} / \mathrm{s}$. Dessa forma, em uma situação hipotética em que o dispositivo fosse empregado para a conversão de energia cinética do escoamento em energia elétrica, a razão $W_{\mathrm{e}} / W_{\mathrm{g}}=0,7$ seria recomendada em detrimento da razão extrema $W_{\mathrm{e}} / W_{\mathrm{g}}=1,0$.

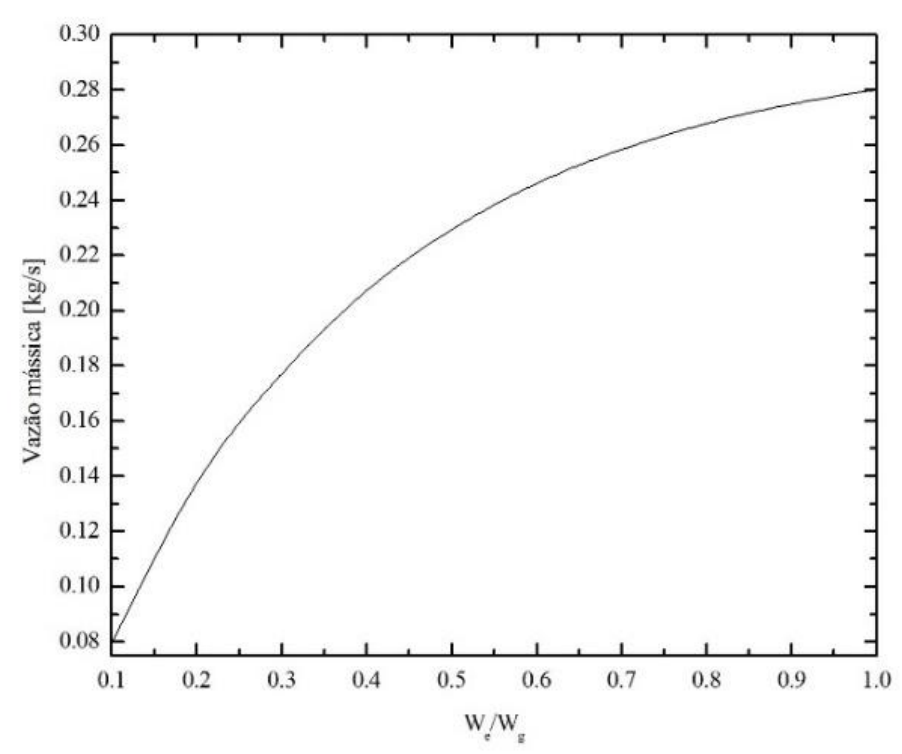

Figura 7 - Efeito da razão $W_{\mathrm{e}} / W_{\mathrm{g}}$ sobre a vazão mássica de ar na saída da chaminé. 


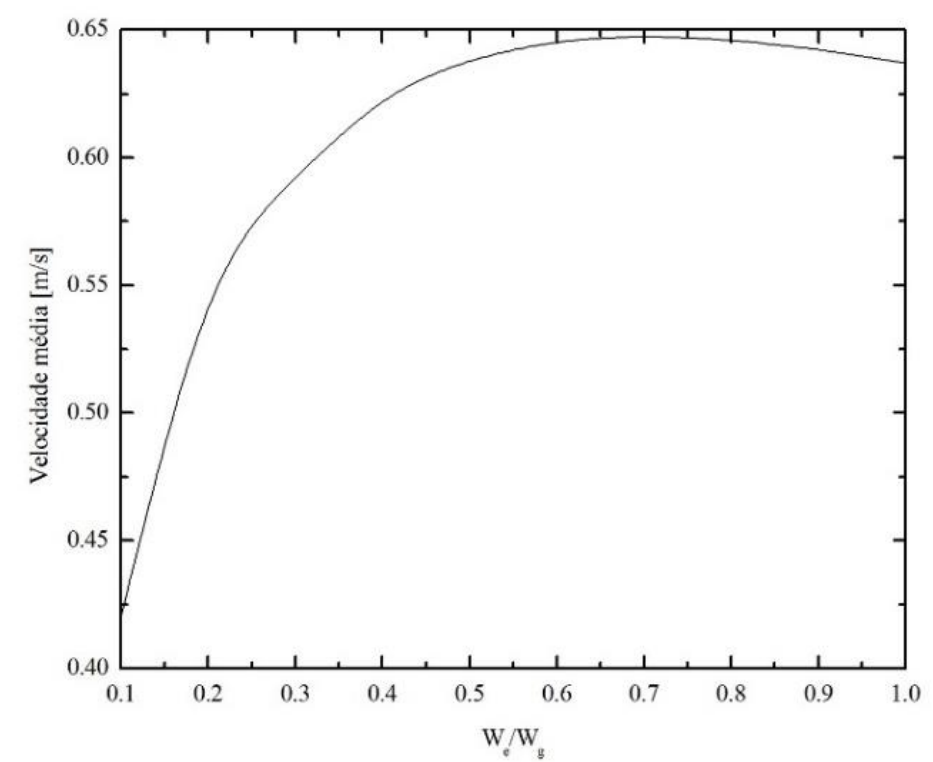

Figura 8 - Efeito da razão $W_{e} / W_{g}$ sobre a velocidade média na saída da chaminé.

Uma causa provável para a vazão mássica ser maior no extremo superior consiste no fato das menores razões estrangularem a seção de saída da chaminé, impedindo que uma maior quantidade de massa passe pela chaminé e, consequentemente, pelo ambiente construído A Figura 10 mostra um perfil de intensidade de turbulência, com relação $W_{e} / H_{t}=$ 0,1 sendo a figura 10 a o pior resultado e com relação $W_{e} / W_{g}=0,1$ e a figura $10 \mathrm{~b}$ o melhor resultado com relação $W_{e} / W_{g}=1$.

Para os campos de velocidade a figura 11 mostra os dois perfis, com relação $W_{e} / H_{t}=$ 0,1 sendo a figura 11 a o pior resultado e com relação $W_{e} / W_{g}=0,1$ e a figura $11 \mathrm{~b}$ o melhor resultado com relação $W_{e} / W_{g}=0,7$. 


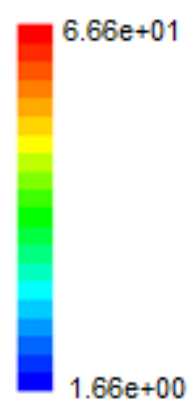

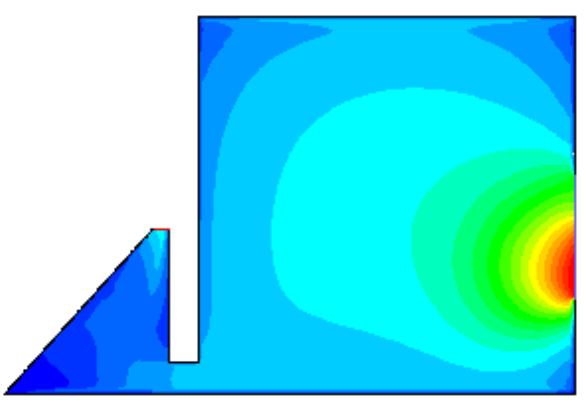

a)

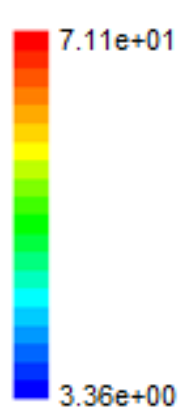

$3.36 \mathrm{e}+00$

b)

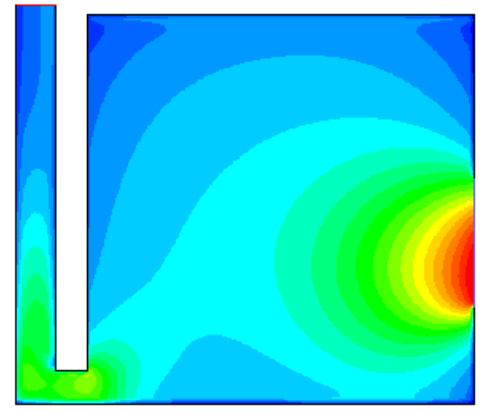

Figura 9 - Campo de intensidade de turbulência para um escoamento a $\mathrm{Ra}_{\mathrm{H}}=1,36 \times 10^{14} \mathrm{e} \mathrm{Pr}$ $=0,71$ para $W_{e} / H_{t}=0,1$ e $W_{e} / W_{g}:$ a) $W_{e} / W_{g}=0,1 ;$ b) $W_{e} / W_{g}=1$ (caso que maximiza a vazão mássica).

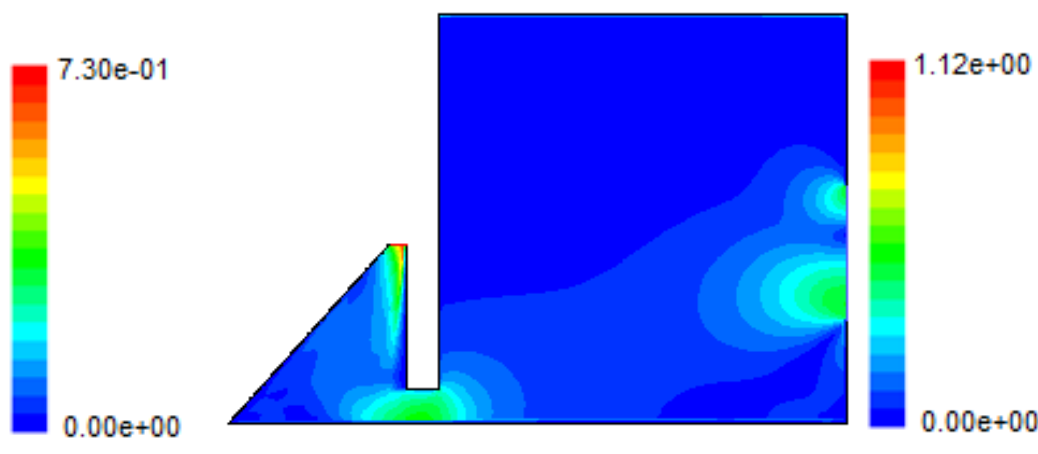

a)

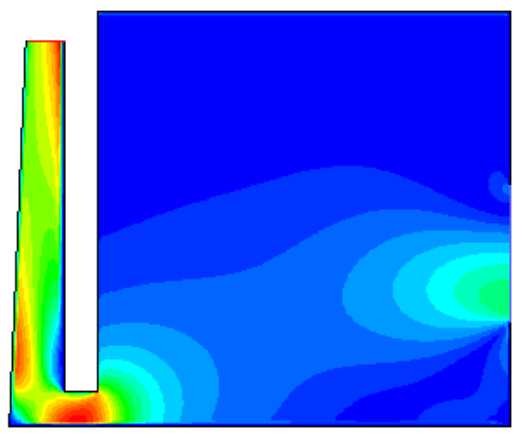

b)

Figura 10 - Campo de velocidade para um escoamento a $\mathrm{RaH}=1,36 \times 10^{14}$ e $\operatorname{Pr}=0,71$ para $W_{e} / H_{t}=0,1$ e $W_{e} / W_{g}:$ a) $W_{e} / W_{g}=0,1$, b) $W_{e} / W_{g}=0,7$ (caso que maximiza a magnitude da velocidade).

\section{CONCLUSÃO}

Foi desenvolvido um estudo numérico com dois objetivos. Primeiramente, desenvolver uma modelagem numérica capaz de reproduzir simulações de uma chaminé solar acoplada a um ambiente construído apresentadas na literatura, mais precisamente no estudo desenvolvido por Khanal e Lei (2015). Posteriormente, com o modelo desenvolvido, foram obtidas recomendações teóricas para a geometria que conduz a maximização da vazão mássica que escoa através do sistema acoplado ambiente-chaminé solar através da aplicação 
do método Design Construtal. Para o modelo numérico proposto, as equações temporais médias de conservação de massa, quantidade de movimento e energia e as equações de transporte do modelo de turbulência foram resolvidas com o método dos volumes finitos (MVF). Para a modelagem da turbulência, emprega-se a modelagem clássica (RANS) com o modelo $\mathrm{k}-\varepsilon$. Primeiramente, foi realizada uma comparação dos resultados do presente trabalho e de Khanal e Lei (2015) para o caso com parede de vidro com ângulo de $4^{\circ}$, que foi o melhor caso obtido no estudo da literatura. Os resultados mostraram uma boa concordância para a vazão mássica adimensional, vazão mássica e magnitude média do campo de velocidades. O desvio encontrado entre as predições da literatura e do presente trabalho foram em torno de $0,3 \%$, o que recomendam a aplicação do método numérico estudado aqui para futuras recomendações geométricas desse dispositivo. Também foi observado que a solução permanente conduziu a campos qualitativos, especialmente na região do ambiente construído, com grande desvio em comparação aos resultados da literatura. Assim, recomenda-se para esse tipo de simulação o uso dos termos transientes na solução.

Com o modelo desenvolvido, foram obtidas novas recomendações geométricas aplicando-se o método Design Construtal. Mais precisamente, a área da chaminé foi mantida constante, bem como o grau de liberdade $W_{e} / H_{t}=0,1$ e variou-se o grau de liberdade $W_{e} / W_{g}$. Para os casos estudados, foi observado que a vazão mássica aumenta com o crescimento da razão $W_{e} / W_{g}$, obtendo-se a vazão uma vez maximizada de $\mathrm{mm}=0,28 \mathrm{~kg} / \mathrm{s}$ para $\left(W_{e} / W_{g}\right) \mathrm{o}=$ 1,0 . Esse valor é $71,6 \%$ maior do que o obtido para o caso com pior desempenho, $W_{e} / W_{g}=$ 0,1. Também foi observado que a geometria que conduz a maior vazão mássica não é a mesma que maximiza a velocidade média na saída da chaminé, onde a razão $W_{e} / W_{g}=0,7$ conduziu a maior velocidade. Isso indica que para objetivos diferentes os parâmetros geométricos podem ser alterados. Futuros estudos é recomendada a avaliação de outras razões $W_{e} / H_{t}$ para verificar se o efeito da razão $W_{e} / W_{g}$ mantém-se semelhante ao obtido aqui para $W_{e} / H_{t}=0,1$.

\section{AGRADECIMENTOS}

Os autores L. A. Isoldi, L. A. O. Rocha e E. D. dos Santos agradecem ao CNPq pelo apoio financeiro e pela bolsa de produtividade em pesquisa. 


\section{REFERÊNCIAS BIBLIOGRÁFICAS}

BASSIOUNY, R.; KORAH, N. S. A.; Effect of solar chimney inclination angle on space flow pattern and ventilation rate. University Department of Mechanical Power Engineering and Energy, 2008.

BEJAN, A.; LORENTE, S.; The Constructal law of design and evolution in nature. Durham: Departament of Mechanical Engineering and Materials Science, Duke University, Laboratory of Materiais and Durability of Construction, University of Toulouse, INSA, 2010.

BEJAN, A.; ZANE, J.; Design in nature. Doubleday, USA, 2012.

CAVALCANTE, F. A. M. S.; Chaminé solar como alternativa para incrementar a ventilação natural de espaços internos. Universidade Federal de São Carlos Centro de Ciências Exatas e de Tecnologia São Carlos. Brasil, 2010.

ÇEngEL, Y. A.; GHAJAR, A. J.; Transferência de Calor e Massa. Porto Alegre: AMGH Editora Ltda., 2012.

FLUENT, Documentation manual - FLUENT 6.3.16, 2007.

IMANI, H.; SHAHREZA, A. R.; Experimental and numerical investigation on an innovative solar chimney. Irã: Mechanical Engineering Division, Dehaghan Branch, Islamic Azad University, Isfahan, Iranb Faculty of Mechanical Engineering, University of Kashan, Kashan, Iran, 2014.

IMRAN, A. A.; Induced flow for ventilation and cooling by a solar chimney. Baghdad: Mechanical Engineering Dept., University of Technology, Baghdad, Iraq Electromechanical Eng. Dept., University of Technology, Baghdad, Iraq, 2015.

INCROPERA, F. P.; WITT, D. P.; Fundamentos de Transferência de Calor e Massa. Rio de Janeiro: LTC-Livros Técnicos e Científicos Editora S.A, 1992.

KHANAL, R.; LEI, C. W.; A numerical investigation of buoyancy induced turbulent air flow in an inclined passive wall solar chimney for natural ventilation. Energy and Buildings, 93: 217 - 226. 2015.

PANTAKAR, S. V. Numerical Heat Transfer and Fluid Flow. New York: McGraw Hill, 1980.

REIS, M. N. E.; Fenômenos de Transporte. Pontifícia Universidade Católica de Minas Gerais. Minas Gerais, 2008.

VERSTEEG, H. K.; MALALASEKERA, W.; An Introduction to Computational Fluid Dynamics: The Finite Volume Method. Pearson, 2007.

VIEIRA, R. S.; Avaliação da Influência Geométrica na potência disponível de uma chaminé solar empregando design construtal. Dissertação de Mestrado em Engenharia Oceânica, Universidade Federal do Rio Grande. Rio Grande, 2015. 
ZAMORA, B.; KAISER, A. S.; Numerical study on mixed buoyancy-wind driving induced flow in a solar chimney for building ventilation. Universidade Dpto. Ingeniería Térmica y de Fluidos, Universidad Politécnica de Cartagena. Cartagena, 2010. 